NIST

PUBLICATIONS

The Measurement of the

Dielectric Constant of

Polymeric Films at High

Fields

\section{Frederick I. Mopsik \\ Brian Dickens}

U.S. DEPARTMENT OF COMMERCE Technology Administration

National Institute of Standards

and Technology

Materials Science and Engineering Laboratory

Polymers Division

Gaithersburg, MD 20899

Prepared for:

Defense Nuclear Agency

Alexandria, VA 22310-3398

Q6C 



\title{
The Measurement of the Dielectric Constant of Polymeric Films at High Fields
}

\author{
Frederick I. Mopsik \\ Brian Dickens
}

U.S. DEPARTMENT OF COMMERCE Technology Administration National Institute of Standards and Technology

Materials Science and Engineering Laboratory

Polymers Division

Gaithersburg, MD 20899

Prepared for:

Defense Nuclear Agency

Alexandria, VA 22310-3398

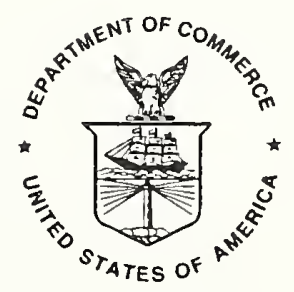

U.S. DEPARTMENT OF COMMERCE Barbara Hackman Franklin, Secretary

TECHNOLOGY ADMINISTRATION

Robert $M$. White, Under Secretary for Technology

NATIONAL INSTITUTE OF STANDARDS

AND TECHNOLOGY

John W. Lyons, Director 


,




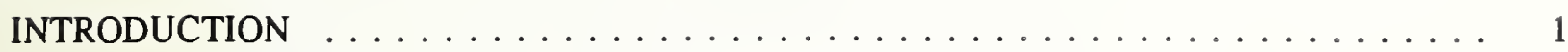

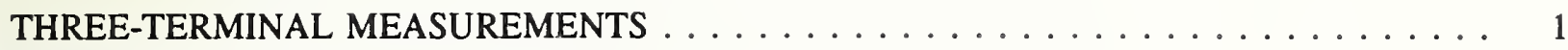

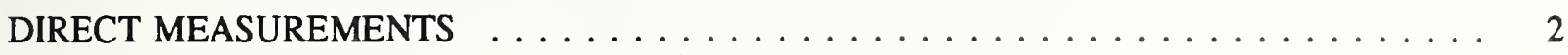

NOISE REQUIREMENTS $\ldots \ldots \ldots \ldots \ldots \ldots \ldots \ldots \ldots \ldots \ldots \ldots \ldots$

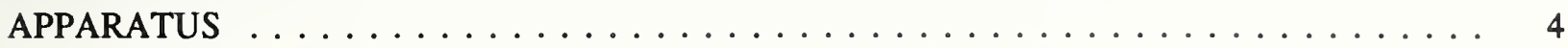

GENERATOR $\ldots \ldots \ldots \ldots \ldots \ldots$

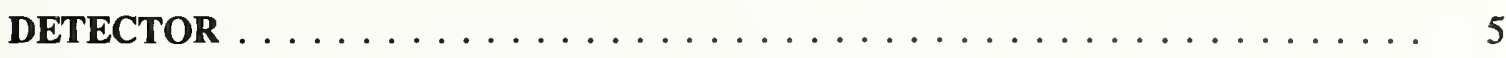

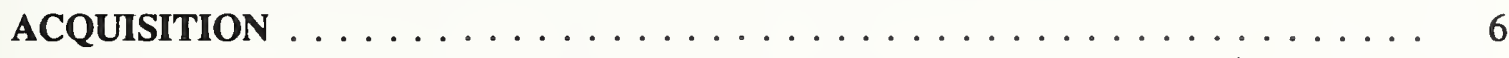

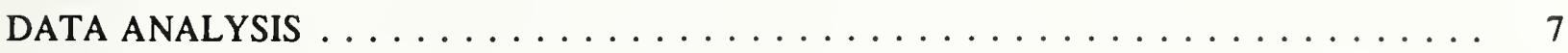

ERROR ANALYSIS $\ldots \ldots \ldots \ldots \ldots \ldots \ldots \ldots \ldots \ldots$

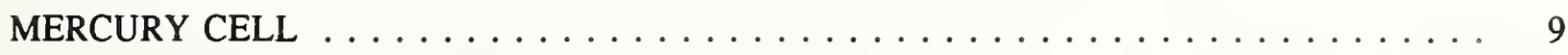

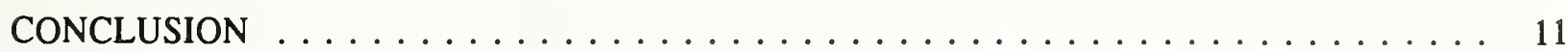




\section{THE MEASUREMENT OF THE DIELECTRIC CONSTANT OF POLYMERIC FILMS AT HIGH FIELDS}

Frederick I. Mopsik

Brian Dickens

Polymers Division

NIST

\section{INTRODUCTION}

The measurement of the dielectric properties of any material requires measuring the current flowing through a sample for a known applied voltage. While this requirement is not apparent when bridge measurements are being used, in recent years it has become more explicit. As an example, the Time-Domain Dielectric Spectrometer ${ }^{1}$ applies a known voltage step to a sample, measures directly the charge flowing across the sample as a function of time and then transforms the data into the frequency domain.

When viewed in this manner, any dielectric measurement becomes limited by two key factors. One is how well a given electrical quantity, such as voltage or capacitance, can be quantified. The other one, usually less appreciated, is how well the measured parameters apply specifically to the sample. Both factors can become difficult as conditions become more extreme relative to conventional measurement techniques.

In this report, we describe a method to measure the dielectric constant of polymeric insulation at high applied electric fields. The method allows the use of shielded specimens to minimize any effects of the surroundings, as well as reduce any personnel hazards. By design, any electrical breakdowns across the sample will not damage the measurement circuitry. Explicit error terms are evaluated in terms of the measurement parameters.

As an illustration, a sample holder using mercury electrodes is evaluated, demonstrating both the reproducibility of the measurements as well as the limitations of the holder. The precision is better than $1 \%$ for films $12 \mu \mathrm{m}$ thick. This precision is sufficient to estimate the physical distortions of the electrode area of the holder upon application of the applied electrical field.

\section{THREE-TERMINAL MEASUREMENTS}

One of the major problems in measuring the impedance of a circuit element is to define the measurement point such that only the desired impedance is included in the measurement. For dielectric measurements, this means being able to measure the capacitance and equivalent parallel conductance of a sample between a pair of electrodes that enclose the sample without any influence on the measurement from the connecting leads or surroundings. Since the sample must be placed at the end of leads to allow for manipulation and temperature control, it is desirable in most cases to try to use a three-terminal measurement configuration. 
In a three-terminal measurement, the leads connected to the two measurement electrodes are shielded and connected to ground. If the measuring circuit is sensitive only to the direct impedance between the two shielded leads and not any shunt impedances to ground, a proper three-terminal measurement is carried out. The main assumption is that the lead impedances in series with the unknown are small enough so that they are negligible. For reasonable values of capacitance (less than $10000 \mathrm{pF}$ ), not too high a $\tan \delta$ (less than 10), frequencies below $1 \mathrm{MHz}$ and ordinary coaxial cables, the conditions are easily met.

The presence of guard rings in a three-terminal measurement is not required, although easily implemented if desired. If a sample with electrodes is simply placed in a shielded box that is sufficiently large, except for the elimination of outside perturbations, a three-terminal measurement would give the same results as an ideal, leadless two-terminal measurement. However, if a grounded electrode, the guard, surrounds one of the electrodes, which is maintained at ground potential, either at bridge balance or by other means, then the measurement becomes guarded. The electric field in the sample is affected by the presence of the guard ring and the final answer could be affected. Any surface currents are intercepted by the guard ring and there can be a reduction in the effective fringing field uncertainty. In a three-terminal measurement, this guard condition is simply achieved by connecting the guard electrode to the grounded enclosure. In this report, both guarded and unguarded measurements were used.

\section{DIRECT MEASUREMENTS}

Classical three-terminal measurements have been associated with the Wagner Earth and transformer bridge measurements. ${ }^{2}$ More recently, a highly precise time-domain method, the TimeDomain Spectrometer ${ }^{1}$ used direct measurements of voltage and charge such that results equivalent to more conventional techniques were obtained.

For direct measurements of a three-terminal impedance, the voltage applied across the sample must be determined and only the charge flowing through the sample must be measured. If both requirements are satisfied with the use of shielded leads, then three-terminal measurement requirements are met.

The determination of the voltage across the sample can be made remotely if the effective output impedance of the voltage source, including the leads, is low enough so that any variation in load does not change the output. For modern power supplies using negative feedback, this condition is well met as long as the total output current is below a critical value. Any shunt capacitance to ground from the high potential lead does not affect the measurement if it does not result in the output capabilities of the supply being exceeded.

The measurement of the charge flowing through the sample must be handled differently. The voltage across the sample must be that of the power supply, if the measurement is to be well defined. As a result, the measurement of the charge must be made such that the current flowing in the measuring circuit does not create a significant voltage drop in series with the sample and voltage source. In Figure 1, this is achieved by using a high gain operational amplifier at the input of the detector such that the sample current and amplifier output current are required to sum to zero by the circuit. Using available circuits, the maximum voltage uncertainty can be held to much less than $1 \mathrm{mV}$, relative to ground. 
If this zero-voltage condition is maintained, then any capacitance to ground between the amplifier input and any shields cannot induce any significant current flow. Therefore, these shunts again do not affect the measurement and a true three-terminal measurement is carried out. It should be mentioned, however, that the shunt low-frequency impedances still must be high since there are amplifier offsets and shunts with too low an impedance can create large errors. In practice, the shunt impedance of most shielded cable is quite acceptable, using good quality amplifiers, even if the shunt capacitance is equal to that of the sample.

\section{NOISE REQUIREMENTS}

Not only must stray impedances be eliminated, but the data must be acquired accurately. Circuit noise must not become large relative to the measured quantities. When the input impedance, as represented by the sample, becomes large and capacitative, input-stage noise can become a major problem.

The ideal input stage to measure current has no parallel impedance to ground so that all the input current flows through a reference circuit element. A zero-input-voltage, current-to-voltage stage typically uses a very high gain operational amplifier such that the input lead is maintained at ground potential by causing an opposing output current to flow through a feedback resistor. All sources of noise, whether from the input stage itself or from the power supply, can be easily represented as sources in series with the sample.

These noise sources are independent of the ideal sample current and see the sample as an impedance in series with the input. For an ideal operational amplifier, the noise gain at any frequency, $G(\omega)$, can be expressed as

$$
G(\omega)=1+\frac{Z_{f}}{Z_{i}},
$$

where $Z_{i}$ and $Z_{f}$ are the input and feedback impedances. In a current amplifier with input capacitance, $C, Z_{f}=R_{f}$ and $Z_{i}=1 / i \omega C_{i}$. For a given value of noise input, the amplifier will have an output proportional to frequency. Since thermal noise, which is the ultimate limit on an experiment, has a constant amplitude for a fixed bandwidth, ${ }^{3}$ there will be an increase of the observed noise with increasing frequency.

The noise problem can be further illustrated by taking the inverse Laplace transform of the right hand side of Eq. 1, which becomes the derivative operator. If the noise signal is replaced by any arbitrary input, such as the output of the generator, the output of the detector is the derivative of that signal, within the bandwidth limitations of that stage. Any irregularities in the input signal will be enhanced, as the derivative accentuates irregularities, and, if they are synchronous with the desired response, will be very difficult to remove as signal averaging will not work.

Rather than trying to overcome these difficulties by elaborate filtering schemes, one can minimize them by using a feedback element that makes the input stage gain more nearly constant with frequency. This is easily done by replacing the feedback resistor with a capacitor so that for an ideal capacitance 
input, the gain is constant. The detector now can be recognized as an ideal charge amplifier. If the normal difficulties of using such a detector, such as drift and overload, can be overcome, the noise problem will clearly be minimized.

There are further benefits from using such a detector. For a capacitive sample, even for a jump in the applied voltage, the output of the detector is bounded by the change in generator output. If the generator produces a ramp, the output follows the input and is not a function of the ramp rate, unlike a current detector. For a given sample capacitance and maximum generator output, the detector response is bounded, no matter what the signal. For a real amplifier, this minimizes range changes and speed requirements.

As a consequence of the benefits of using a charge amplifier, the requirements on the design of the input stage can be more readily optimized. The energy storage inherent in the feedback capacitor allows steps to be completely captured with amplifier responses not needed to be any faster than the excitation. The overall gain does not need to be scaled to the time scale of the measurement, as it must with a current amplifier. Gain changes are minimized, greatly facilitating experimental design. As will be discussed later, a suitable charge amplifier can be designed that not only reduces experimental noise, but also is immune to any sample breakdown.

\section{APPARATUS}

The general design of the high-voltage dielectric constant system consists of a programmable, low output impedance generator, a zero input voltage charge amplifier with a computer to provide control of the generator and amplifier, carry out the data acquisition and perform data analysis. There is enough flexibility in the system so that the measurement can be carried out for a wide range of waveforms.

\section{GENERATOR}

The generator consists of a high voltage, high slew rate, bipolar amplifier driven by a suitable programmable function generator. The amplifier that we used could achieve $10 \mathrm{kV}$ at 2 ma with a slew rate of $30 \mathrm{~V} / \mu \mathrm{s}$. Voltage regulation was better than $0.1 \%$ within the maximum current limit. At the settable current limit, there was a fold-back limit circuit and trip point. The supply had a voltage monitor output with a 1000:1 output ratio that we calibrated with a precision divider and found to be accurate to better than $0.1 \%$. This monitor output was used by the acquisition system to measure the actual output voltage.

The amplifier, which had a fixed gain of 1000 , was driven by a function generator with an IEEE488 interface. The particular one that we used could generate only fixed waveforms with offsets. For energy storage applications at high field, it was programmed to generate a single, negatively going triangular pulse. Prior to the pulse the offset was increased by the computer over several seconds until the desired maximum voltage was achieved. After a suitable period of time, a pulse was initiated such that the voltage decreased to zero and then returned to the offset. The negative going ramp was used to determine the recoverable discharge energy. 
This generator setup is being updated for increased versatility. In particular, an arbitrary function generator is being obtained in which up to 65000 points can be used to define any desired waveforms. With this generator, almost any charging and discharging waveform can be employed to study the behavior of the sample. The main limits to the excitation are set by the amplifier, which is also being upgraded.

In addition to limits set by the frequency response of the high voltage amplifier, the rate limitation on the change in applied voltage can be set by the current capability of the amplifier for capacitive samples. Just as the large currents induced by rapid voltage changes create problems for a current detector, they also can create problems for the generator which has to supply those currents. For a given capacitance, the current required to change the voltage at a rate $d V / d t$ is given by $I=C(d V / d t)$. For many samples, the amplifier's current limit is smaller than the current that would flow through the sample at the amplifier's maximum slew rate. In these instances, the maximum rate of change is set by the current limit of the high voltage supply.

\section{DETECTOR}

The other key item is the charge detector. Not only must it not show appreciable output drift during the time required for a measurement, but it must also be able to withstand any possible breakdowns of the sample. The design that was developed is shown in Figure 2. A low bias current operational amplifier is used with a capacitor as a feedback element. The amplifier has a bias current of less than $1 \mathrm{pA}$ with a offset voltage drift of less than $5 \mu \mathrm{V} /{ }^{\circ} \mathrm{C}$. The feedback capacitors are polypropylene film capacitors that can be considered ideal and lossless for the accuracies required in these measurements. The reset switch was a low leakage reed relay to minimize drifts from voltages that can occur across it.

Unlike the detector developed for the Dielectric Spectrometer, ${ }^{1}$ the leakage and bias currents are not as critical for the one required for high-field measurements. As long as low bias current operational amplifiers are used and good polymeric insulation used in its construction, the total charge generated by the stray currents will be much smaller than the total charge transferred by the sample. For a $1000 \mathrm{pF}$ sample charged to $5 \mathrm{kV}$, the total charge is $5 \mu \mathrm{C}$. With a total leakage current of $10 \mathrm{pA}$, which is readily obtainable with good practice, even a $100 \mathrm{~s}$ measurement will have a drift of less than $0.1 \%$ of the total charge involved. For comparison, the bias current of $0.03 \mathrm{pA}$ achieved in the Dielectric Spectrometer required careful construction with extremely low leakage insulation.

A more important consideration in the design is protecting the amplifier, which can only sustain about $10 \mathrm{~V}$ on the input. This too was a problem for the Time Domain Spectrometer and, as for the leakage requirements, the solution is somewhat eased by the larger current levels involved. Since the full scale output of the detector is $10 \mathrm{~V}$, full scale referred to the input is set by the value of the feedback capacitor. In order to achieve a full scale of $10 \mu \mathrm{C}$, the feedback capacitor is increased from $1 \mathrm{nF}$ in the Spectrometer to $1 \mu \mathrm{F}$. As the capacitor connects the input to the low impedance output, it can act as a shunt if the response of the amplifier cannot follow the input. This larger capacitance helps attenuate the maximum possible applied voltage, especially if the current limit of the generator amplifier is considered. 
The detector is further protected by a pair of JFET transistors connected as voltage limiters, as shown in Figure 3. When the maximum gate to source voltage is exceeded $(<1 \mathrm{~V})$, they will conduct and act as voltage clamps, easily tripping the generator's preset current limiter. The current limiting resistor is inside the feedback loop and does not contribute to the detector's response. This resistor should be sized to withstand the maximum voltage that could be across it, subject to the constraint that the current through the JFETs should be limited to less than $100 \mathrm{~mA}$. In our detector, the limit was set by the output amplifier's current limit rather than the maximum output voltage. In combination with the damping contributed by the feedback capacitor, the detector has not been affected by sample breakdowns.

An unexpected limitation that was encountered in the use of the detector was the ability of the detector to sink a discharge current with a fast ramp. In this case, the current, while less than the rated detector output, is opposite in sign to the output voltage. Therefore, the current must be supplied by the output transistor connected to the opposing supply and, hence, the transistor will experience a voltage equal to a supply voltage plus the maximum output voltage. Under this circumstance, an undocumented secondary breakdown can occur in the output stage. While it does not destroy the detector, it adds noise to the desired signal, just as if the sample were breaking down. The main difference is that this "noise" is very reproducible and starts to occur at a well defined ramp rate for a given output. The solution is either to use a buffer amplifier between the detector amplifier and the feedback capacitor or else to use a high output amplifier. Since they were available, we used a FET input amplifier with $1 \mathrm{pA}$ bias current and capable of sinking $100 \mathrm{~mA}$ at $10 \mathrm{~V}$ or $50 \mathrm{~mA}$ at $100 \mathrm{~V}$, Apex Microtechnology PA $08 \mathrm{~A}^{*}$. With this circuit, we did not observe any perturbations due to the amplifier output limitations.

As an extension, if it were required, even this amplifier could be used with a current booster stage. Since there are readily available boosters, this is not considered to be a major concern. Alternatively, an output stage using MOSFETs could be used as they are free of the secondary breakdown problem.

\section{ACQUISITION}

The acquisition system employed was a commercial acquisition board with a 12 bit resolution analog to digital converter (ADC) that could digitize a sample as fast as every $5 \mu$ s into a FIFO memory. Even for a total ramp time of only $1 \mathrm{~ms}$, this would be sufficient time resolution for these measurements. By direct test with voltage standards, the board was found to have a one bit noise level so that the resolution was 1 in 2048 centered at zero volts. The board had an independent clock so that timing was set by a quartz clock rather than by any programming steps. As a result no significant error was associated with the measurement of time.

The board had 8 multiplexed inputs that could be scanned as a burst at a fast rate as a burst with a slower overall sampling rate. The logic allowed sampling four inputs every $5 \mu$ s with any desired spacing between each burst. As a result, the generator output could be measured at nearly the same time as the charge so that no assumptions had to be made as to the signal appearing across the sample. At the

*The naming of commercial products is neither an endorsement nor a recommendation by NIST. The names are only used to completely describe the measurements and do not necessarily represent the best choice for the indicated application. 
rates used in the measurements at NIST, with ramps as short as $10 \mathrm{~ms}$, the measurements can be considered simultaneous.

The sampling rate was determined by setting a delay counter on the acquisition board so that 256 bursts uniformly covered the time for the ramp down and the ramp back up. In addition, 33 bursts were added so that the acquisition board could be triggered somewhat in advance of the generator and both the leading and trailing edges of the measurement could still be included.

\section{DATA ANALYSIS}

The data, which included both the applied voltage and transferred charge for both the down and following up ramps were then analyzed for the total charge transferred during each ramp. The voltage data were scanned for the first maximum, the first minimum and a second maximum. These points were used to define the starting and ending points of the ramps.

The energy for the discharge ramp was then computed as

$$
U_{d i s}=\sum_{i=1}^{i_{0}-1}\left(Q_{i+1}-Q_{i}\right)\left(V_{i}+V_{i+1}\right) / 2,
$$

where $U_{d i s}$ is the discharge energy, $Q_{i}$ is the charge and $V_{i}$ is the voltage for time sample $i$ and $i_{o}$ is the point for which the voltage becomes zero.

For discharge, this energy should be correct, even though there was a following ramp up. For data points prior to the sampling window the current was sufficiently small so that the uncertainty in the starting time was not significant. While for times after $i_{0}$ there could be a significant charge flow, the voltage can be taken as zero, and hence this charge will not contribute to the discharge energy.

Finally, the discharge energy can be normalized to an equivalent dielectric constant. The energy of an ideal capacitor is given by $1 / 2 C V^{2}$. If the sample thickness, $t$, and area, $A$, are known, then the capacitance is given by

$$
C=\frac{\epsilon \epsilon_{0} A}{t},
$$

where $\epsilon_{o}$ is the permittivity of free space. Combining these expressions gives for the effective dielectric constant, $\epsilon$, 


$$
\epsilon=\frac{2 U_{\text {dis }} t}{\epsilon_{0} A V^{2}} .
$$

From Equation 4, it can be seen that both the electrode area and sample thickness enter directly into the computed $\epsilon$. For a sample holder such as the mercury cell described later in this report, the electrode area is relatively constant from sample to sample. The thickness, however, is not. For accurate work each sample must be measured. In this laboratory, the thickness of each sample was individually measured using a thin-film two-fluid cell. ${ }^{4}$

\section{ERROR ANALYSIS}

The main limits to the accuracy, aside from limits set by the sample and cell, are set by linearity in the circuits, calibration errors, rate limitations, and noise. In the system described in this report, linearity and noise are the main factors.

Linearity is limited by the ADC to 1 part in 2048 and is inherent to the device. This error, which is normally quite small, can be increased due to experimental requirements. In particular, the range of the measured parameters is always smaller than full scale. In our original setup, the output voltage and charge must be measured on the same full scale acquisition range. While the charge amplifier has several selectable range capacitors, at low voltage values there still could be some increase in error for low values of the total charge.

Calibration errors of the electronics were for the most part much less than $0.1 \%$. The output monitor of the amplifier was calibrated using a precision divider probe with digital voltmeter that had been calibrated to $0.01 \%$. Similarly, using a variable supply, the ADC was calibrated with the same digital voltmeter. The feedback capacitors were calibrated using a digital oscilloscope, a reference capacitor and a $100 \mathrm{~V}$ step generator calibrated with the same voltmeter. The estimated uncertainty for the 0.1 and $1 \mu \mathrm{F}$ feed back capacitors in the detector was $1 \%$ and was verified with a precision transformer bridge. The uncertainty in the $10 \mu \mathrm{F}$ capacitor was several times larger due to noise limitations in the oscilloscope. Differential measurements could have been made so that all desired quantities were traceable to the same voltmeter, but this was not considered necessary.

As can be seen, the overall uncertainty is about $1 \%$. If ultimate accuracy were desired, relating all dielectric parameters to one common voltmeter would limit the calibration error limited to the voltmeter's linearity, since all measurements could be reduced to voltage ratios. As the actual limits to measurement accuracy were set by sample to sample variation, unknown time response errors and sample holder limitations, no attempt was made to further reduce the electrical measurement calibration error.

The only remaining consideration in calibration is the error due to response limitations in the electronics. Since the output of the generator is directly tracked by circuitry that is much faster than the output amplifier, the only possible contribution to the measurement uncertainty from limitations in the generator would be from applying a nonideal waveform. This error should be quite small. 
The more important dynamic error source is that of the detector. A current amplifier could have large errors due to lost amplitude from either rate limitations or actual clipping. However, in a charge amplifier, any delayed response must store charge in the feedback capacitor. As long as the amplifier input voltage does not get high enough to turn on the JFET protection circuit, the output must eventually follow the total charge flow. Therefore, there can only be a somewhat delayed response but of correct total amplitude.

If this were a simple measure of dielectric constant, at long times, or low frequencies, the correct answer would still be obtained. For a measurement of energy where the product of charge transferred and applied voltage is required, there will be an error that is a function of the charge amplifier response, although clearly much smaller than that for current amplifier. In the system constructed at NIST, the detector had a frequency response of $30 \mathrm{MHz}$ and a slew rate of $30 \mathrm{~V} / \mu \mathrm{s}$. For ramps of $10 \mathrm{~ms}$ duration or greater, the dynamic error is not expected to be significant.

The last source of measurement error to be considered is that due to noise. For the system described in this report, the main source of noise, aside from digitization, was the function generator at a level of about $0.1 \%$ of full scale. This error could be reduced with a better generator and will be in the near future. However, for most measurements made to date, the limits to experimental precision were due to the digitization error of about 1 bit and the time dependent behavior of the sample and its holder. For a low loss material, such as polypropylene, replicate measurements could be made to within $1 \%$. The absolute accuracy was set more by the variation of film thickness than any other source of error.

\section{MERCURY CELL}

The sample holder that has been used for the measurements at high voltages employed a mercury pool to form contacting electrodes. This cell ensured good electrical contact as well as a lack of sharp edges on the electrodes. The pool was confined between sheets of poly(methyl methacrylate), PMMA, spaced so that a polymer film could be inserted in the center, dividing the pool in half to form both electrodes. Connection was made by iron needles brought out at opposite sides of the cell. The mercury was retained by surface tension. A cross section of the holder is shown in Figure 4.

The holder has fully contacting electrodes without requiring any metallization. Provided fresh, clean mercury is used, the electrode corners have a natural curvature so that the tendency for electrical breakdown at the edges is greatly reduced. The sample can be inserted into the holder by sliding it into a slot dividing the halves of the PMMA walls. Also, if the film is large enough, fresh portions of the sample can readily be moved between the electrodes.

The cell does suffer from some limitations. One is that there is some coupling between the two mercury electrodes through the PMMA. This coupling can be from both the stray fringing fields at the outside edges of the mercury pools and the iron connecting pins.

Also, the cross-sectional area is somewhat uncertain. There is no area-defining guard ring. The fringing field is influenced by the difference in dielectric constant between the film and the PMMA. Finally, the area of contact could be a function of film thickness and sample gap between the PMMA and the film to be measured. There could even be a voltage dependence as a result of the electrostatic attraction between the mercury pools at high applied field strengths. 
In order to minimize any effects from these possible sources of error, a calibration set of measurements was made using commercial polypropylene, PP, poly(ethylene terephthalate), PET, and polyvinylidene fluoride, PVDF, films. These films were approximately $12 \mu \mathrm{m}$ in thickness. Each film was individually measured for thickness and dielectric constant in a thin-film, two-fluid cell at NIST. ${ }^{4}$

To estimate any significant contribution to the measured capacitance by the fringing fields, these films of differing dielectric constant were used to compute the effective area of the mercury cell. Since the two-fluid cell electrode area was larger than that of the mercury cell, ten capacitance readings were taken in the mercury cell over the portion of the film that was measured in the two-fluid cell. The average value was then used to calculate the effective area of the mercury cell using the measured thickness and the dielectric constant of each individual film. At the low fields used in these measurements, the effective area, $2.90 \mathrm{~cm}^{2}$, was found to be a constant for all films to within measurement uncertainty of $0.02 \mathrm{~cm}^{2}$. This constancy for films of this thickness shows that fringing fields were not significant.

Another set of measurements was used to estimate time dependence as well as voltage dependence of the cell. Measurements were made on two PP and one PET films, all $12 \mu \mathrm{m}$ thick, at voltages up to $5 \mathrm{~K}$ in increasing steps of $500 \mathrm{~V}$ or until electrical breakdown. Another PP film, with metallized electrodes of constant area, was measured in another sample holder over the same voltage range. At each voltage step, a series of five readings were taken. For all the films measured in the mercury cell, the values for $\epsilon$, averaged over all voltages, as a function of measurement step were constant to within a measurement standard deviation of less than $0.5 \%$. Since all measurements were made with the same increasing step in voltage, this result indicates no consistent, detectable time-dependent process on the time scales employed in these measurements. This result is consistent with the effective area being constant as a function of film $\epsilon$. For films of these thicknesses it indicates that any stray fields in the PMMA holder are of negligible importance.

However, when the same data for the $\mathrm{Hg}$ cell, averaged over all runs at a given voltage, are analyzed as a function of voltage,the results are quite different. The data, displayed as values reduced by the average value at $2.5 \mathrm{kV}$, are shown in Figure 5 . The heavy line is the least squares straight line fit to all the data. Except for some low voltage points where noise was a larger problem, the data fit the line to within $0.5 \%$, showing the consistency of the data. Also shown, displaced along the vertical axis for clarity, are data for the PP that was measured using thin evaporated aluminum electrodes.

The slope of the line for the mercury cell data is $0.0120 \pm 0.0008 \mathrm{kV}^{-1}$, independent of the film. This voltage dependence could arise from several effects directly attributable to the construction of the cell. At the voltage levels used in these measurements, there are significant mechanical forces on the mercury pool from the electric field. For $12 \mu \mathrm{m}$ film with an $\epsilon$ of 2, there is an approximate pressure of $2.5 \mathrm{MPa}$ at $5 \mathrm{kV}$. This pressure can not only compress the film, but also alter the meniscus of the mercury in the gaps surrounding the measured film. Since the force is attractive, both effects are positive.

The effects can be separated using the metallized electrode data. For the metallized data, there can be no changes present due to the electrode while the forces on the film should be nearly identical to those in the mercury cell. For this case the slope was $0.0051 \pm 0.0009$ indicating that the changes due to the mercury are 2.5 times larger than those due to a change in the film dimension. 
As a last comment, the mechanical forces were sufficiently large so that during the discharge stage the impulse imparted by the rapid ramps displayed an easily observed motion in the mercury if there was any air space in the cell above the mercury pool. While there was no apparent harm made to the measurement, this motion and stress could affect the ultimate breakdown strength of a measured film.

\section{CONCLUSION}

It is possible to design a generic, three-terminal dielectric test system with accuracies of $1 \%$ or better for $\epsilon$. While the system described in this report concerned itself with the apparent dielectric constant upon discharge, other waveforms and data analysis could easily be employed. If the function generator used in this report is replaced with an arbitrary function generator, than many other possible measurements are possible. Furthermore, if needed, the Laplace transform techniques used in the Dielectric Spectrometer could be used. With currently available instrumentation, short time responses approaching $1 \mu$ s are possible. The long time limit is only set by drift and stability considerations. 


\section{References}

1. F. I. Mopsik, Rev. Sci. Instrum 55, 79 (1984).

2. R. H. Cole, P. M. Gross, Rev. Sci. Instrum. 20, 252, (1949).

3. S. O. Rice, "Mathematical Analysis of Random Noise", in "Selected Papers on Noise and Stochastic Processes", N. Wax, ed., Dover Publications (New York, 1954).

4. F. I. Mopsik, "Two Fluid Measurements on Thin Films", NIST Tech. Note 1294, U. S. GPO, Washington, D. C., 1992. 

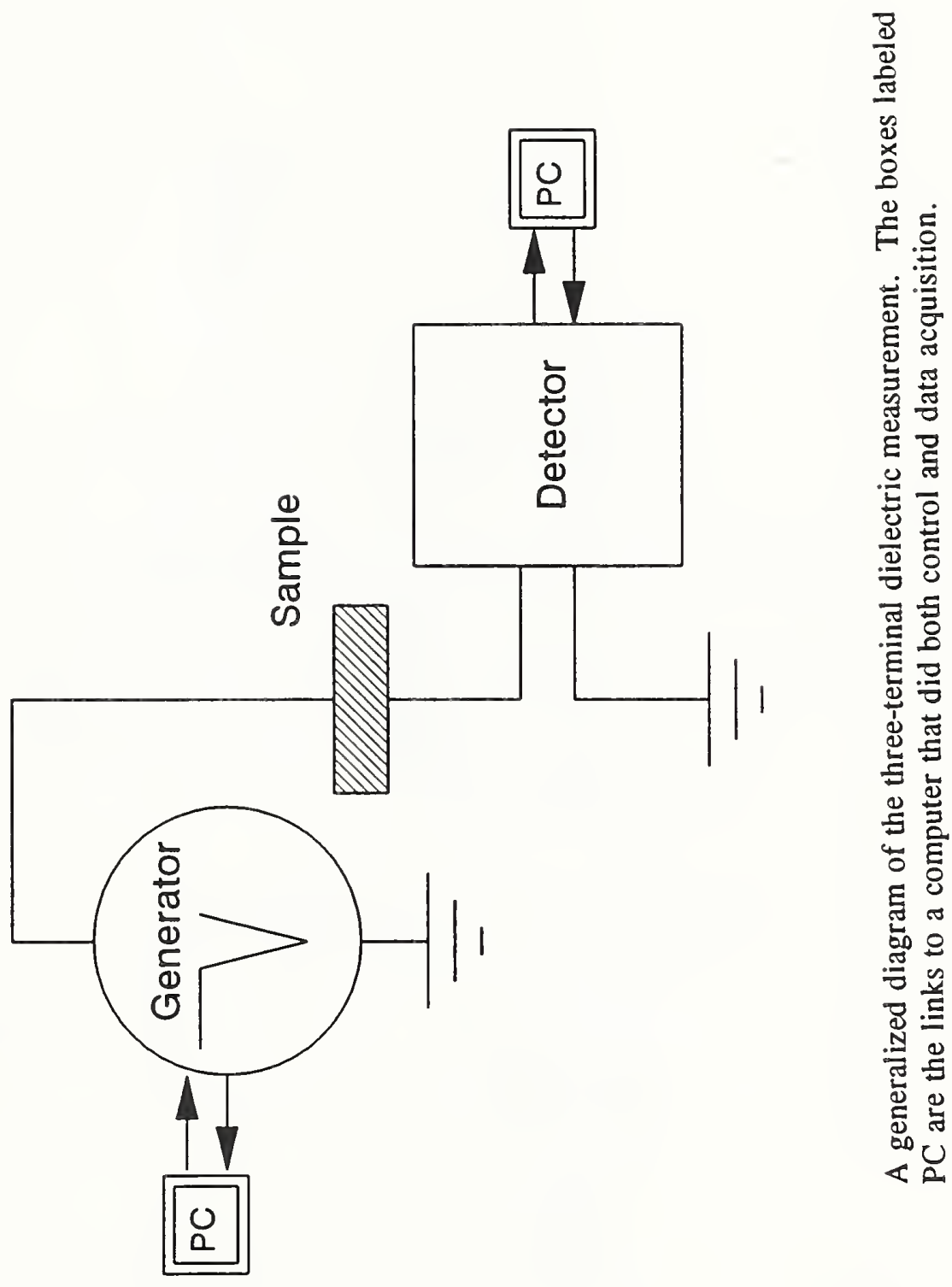

可 

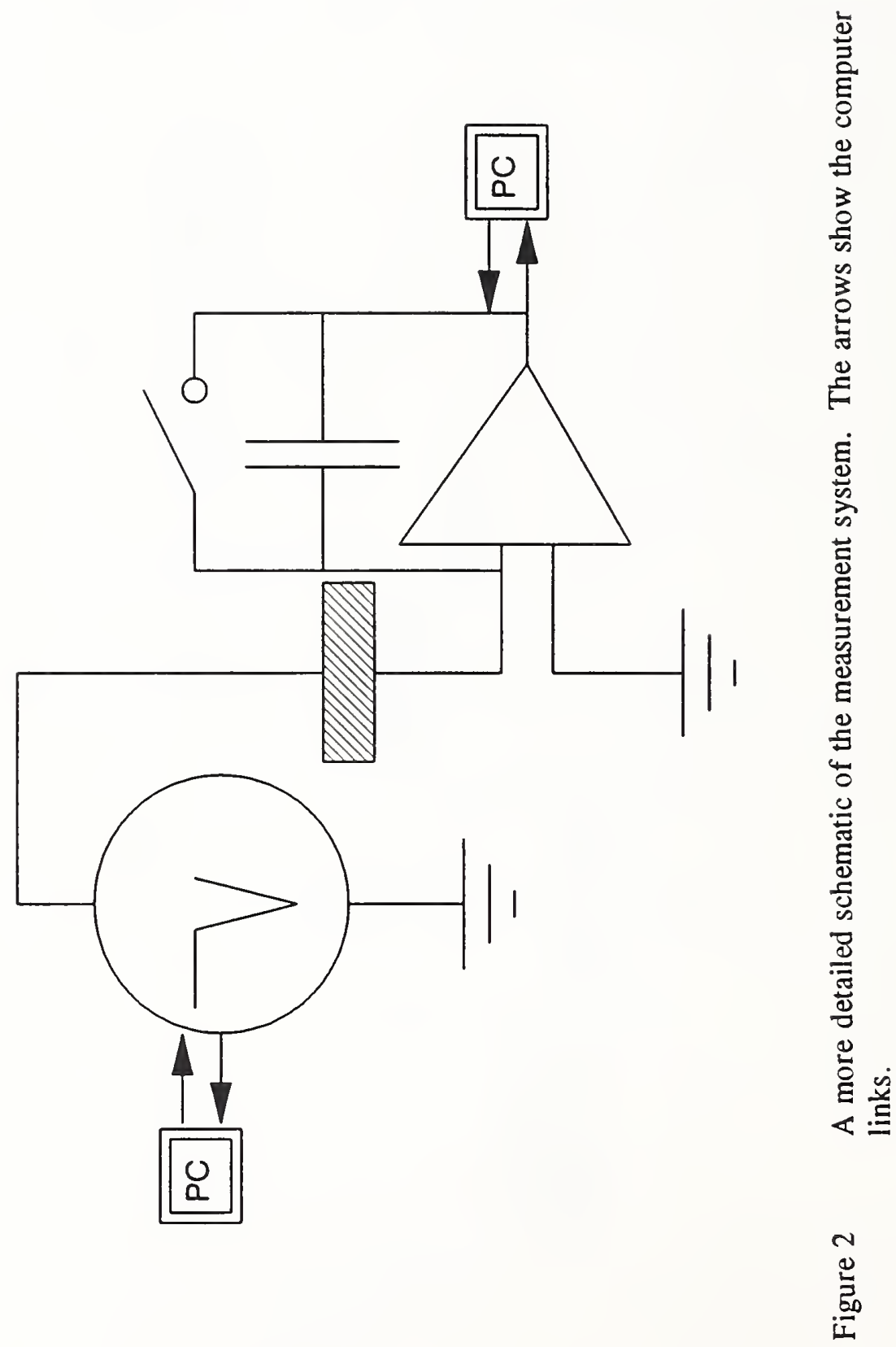


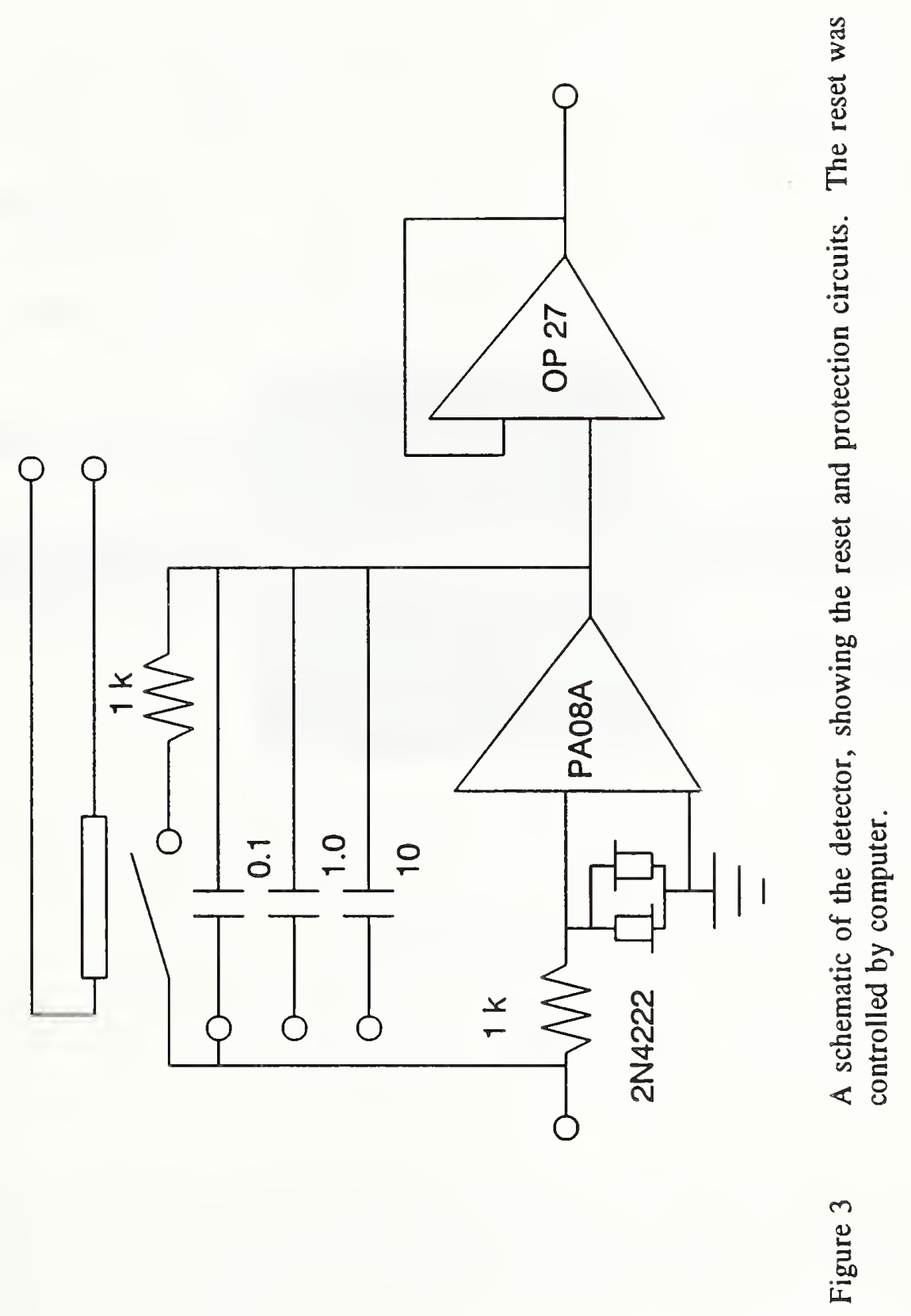




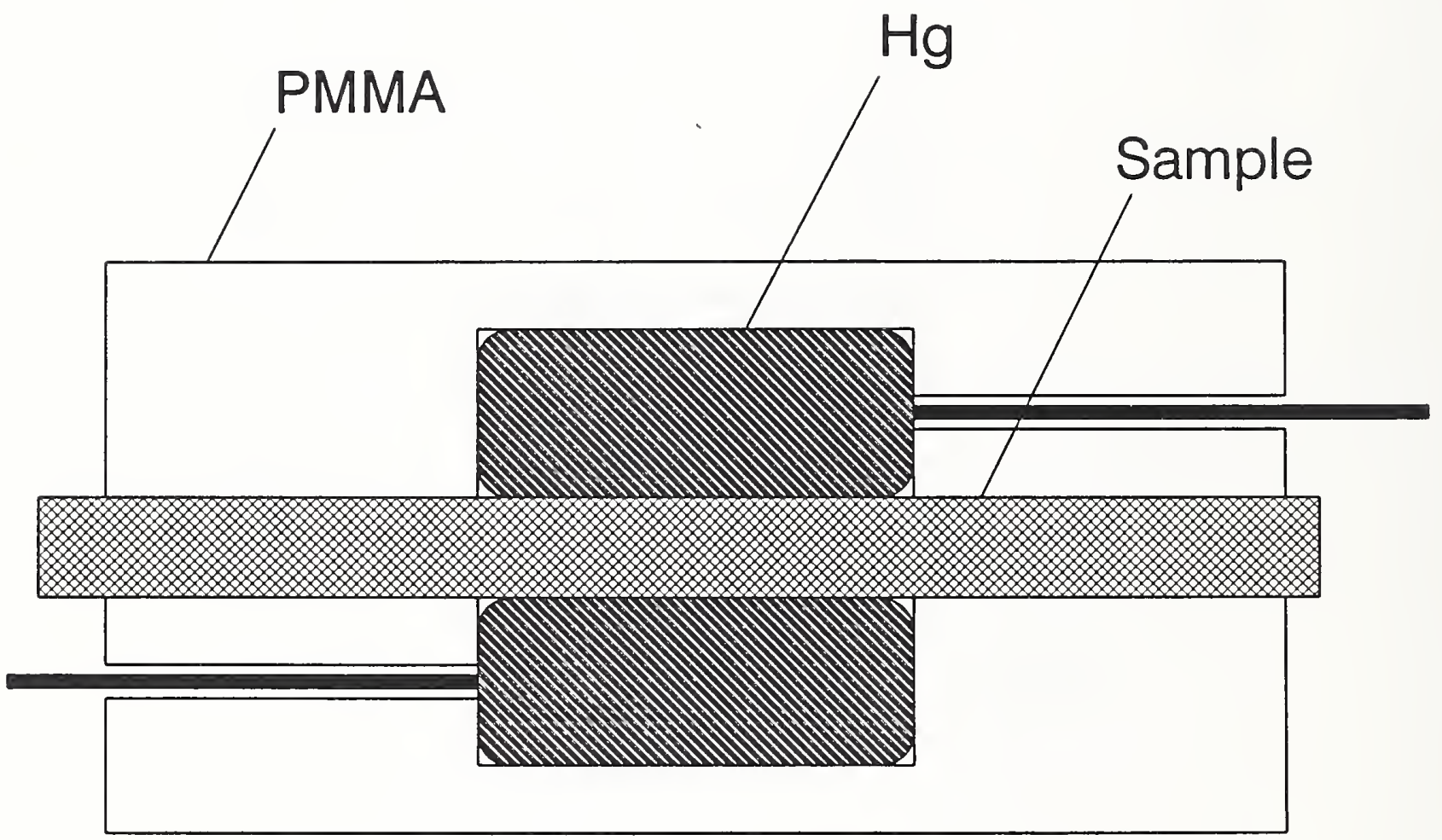

Figure 4 A cross section of the mercury cell. The mercury was held in place by surface tension. 


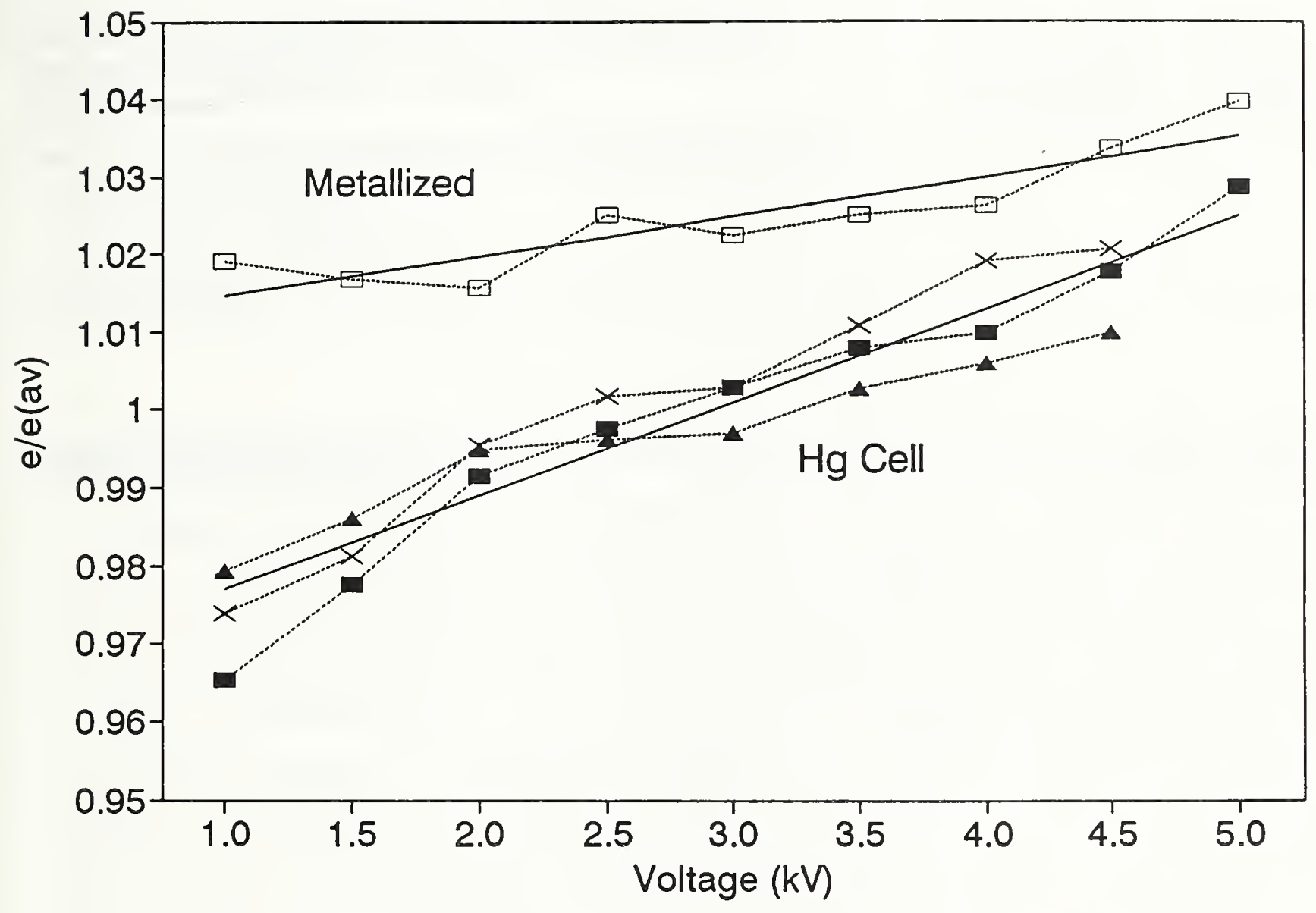

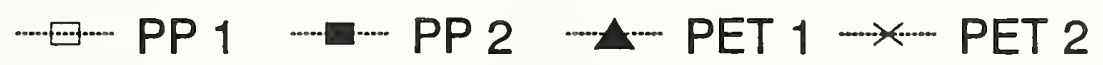

Figure 5 The normalized values of the high field value for $\epsilon$ vs $V$ for PP and PET. The results for the metallized electrodes are displaced vertically for clarity. The broken lines are for connection only while the solid lines are the least squares fits to a straight line for the entire data set for each type of electrode. The difference in slope between the two least squares lines is significant. 


\begin{tabular}{|c|c|c|}
\hline \multirow{3}{*}{$\begin{array}{l}\text { NIST-114A } \\
\text { (REV. 3-90) }\end{array}$} & \multirow{3}{*}{$\begin{array}{l}\text { U.S. DEPARTMENT OF COMMERCE } \\
\text { NATIONAL INSTITUTE OF STANDARDS AND TECHNOLOGY } \\
\text { BIBLIOGRAPHIC DATA SHEET }\end{array}$} & $\begin{array}{l}\text { 1. PUBUCATION OR REPORT NUMBER } \\
\text { NISTIR } 4910\end{array}$ \\
\hline & & 2 PERFORMING ORQANIZATION REPORT NUMBER \\
\hline & & $\begin{array}{l}\text { 3. PUBLCATION DATE } \\
\text { SEPTEMBER } 1992\end{array}$ \\
\hline
\end{tabular}

4. TITLE AND SUBTITLE

THE MEASUREMENT OF THE DIELECTRIC CONSTANT OF POLYMERIC FILMS AT HIGH FIELDS

5. AUTHOR(S)

Frederick I. Mopsik and Brian Dickens

6. PERFORMING ORQANIZATION (IF JOINT OR OTHER THAN NIST, SEE INSTRUCTIONS)

U.S. DEPARTMENT OF COMMERCE

MATIONAL INSTITUTE OF STANDARDS AND TECHMOLOGY

CATHERSBURG, MD 20890

\begin{tabular}{|l|} 
7. CONTRACT/ORANT NUMBER \\
\hline $\begin{array}{l}\text { 8. TYPE OF REPOAT AND PERIOD COVERED } \\
\text { Final Report, Sept. } 1992\end{array}$ \\
\hline
\end{tabular}

9. SPONSORING OAGAMIZATION NAME AND COMPLETE ADDRESS (STAEET, CITY, STATE, ZIP)

Defense Nuclear Agency

6801 Telegraph Road

Alexandria, VA 22310-3398

10. SUPPLEMENTAAY MOTES

11. ABSTRACT (A 20O-WORD OR LESS FACTUAL SUMMAAY OF MOST SIGNIFICANT INFORMATION. IF DOCUMENT INCLUDES A SIGMIFICANT BIBLOGRAPHY OR UTERATUAE SURVEY, MENTION IT HERE)

A generalized method for measuring the dielectric constant of a film at high applied

fields is outlined. By using a low output impedance generator and a zero input voltage charge amplifier, a three-terminal configuration becomes possible. This method allows a complete specimen shielding. It also removes from the measured parameters any influence of the connecting leads. The measurement advantages of using a charge amplifier are discussed. A design for the charge amplifier is given that provides good immunity from any damage if the sample should experience an electrical breakdown. An extensive error analysis of the method is provided. Results are presented that show data with an uncertainty of $<1 \%$ are possible in well defined samples.

12. KEY WORDS (6 TO 12 ENTAIES; ALPHABETICAL ORDER; CAPITALIZE ONLY PROPER MAMES; AND SEPARATE KEY WORDS BY SEMICOLONS)

capacitative input noise; charge amplifier; dielectric constant; electrical

measurements; high voltage; thin film; three-terminal measurements

FOR OFFICIAL DISTRIBUTION. DO NOT RELEASE TO MATIONAL TECHNICAL INFORMATION SERVICE (NTIS).

ORDER FROM SUPERINTENDENT OF DOCUMENTS, U.S. OOVERMMENT PRINTING OFFICE, WASHINOTON, DC 20402.

ORDER FROM NATIOHAL TECHMICAL INFORMATION SEAVICE (NTIS), SPRINGFIELD, VA 22161.

14. NUMBER OF PRINTED PAGES

21

15. PRICE

$\mathrm{A} 02$ 


